\title{
Spatial dispersion in two-dimensional plasmonic crystals: Large blueshifts promoted by diffraction anomalies
}

David, Christin; Christensen, Johan; Mortensen, N. Asger

Published in:

Physical Review B

Link to article, DOI:

10.1103/PhysRevB.94.165410

Publication date:

2016

Document Version

Publisher's PDF, also known as Version of record

Link back to DTU Orbit

Citation (APA):

David, C., Christensen, J., \& Mortensen, N. A. (2016). Spatial dispersion in two-dimensional plasmonic crystals: Large blueshifts promoted by diffraction anomalies. Physical Review B, 94(16), [165410].

https://doi.org/10.1103/PhysRevB.94.165410

\section{General rights}

Copyright and moral rights for the publications made accessible in the public portal are retained by the authors and/or other copyright owners and it is a condition of accessing publications that users recognise and abide by the legal requirements associated with these rights.

- Users may download and print one copy of any publication from the public portal for the purpose of private study or research.

- You may not further distribute the material or use it for any profit-making activity or commercial gain

- You may freely distribute the URL identifying the publication in the public portal 


\title{
Spatial dispersion in two-dimensional plasmonic crystals: Large blueshifts promoted by diffraction anomalies
}

\author{
Christin David, ${ }^{1, *}$ Johan Christensen, ${ }^{1,2}$ and N. Asger Mortensen ${ }^{1,3}$ \\ ${ }^{1}$ DTU Fotonik, Department of Photonic Engineering, Technical University of Denmark, DK-2800 Kongens Lyngby, Denmark \\ ${ }^{2}$ Instituto Gregorio Millán Barbany, Universidad Carlos III de Madrid, ES-28916 Legans (Madrid), Spain \\ ${ }^{3}$ Center for Nanostructured Graphene, Technical University of Denmark, DK-2800 Kongens Lyngby, Denmark
}

(Received 23 June 2016; revised manuscript received 21 September 2016; published 12 October 2016)

\begin{abstract}
We develop a methodology to incorporate nonlocal optical response of the free electron gas due to quantuminteraction effects in metal components of periodic two-dimensional plasmonic crystals and study the impact of spatial dispersion on promising building blocks for photonic circuits. Within the framework of the hydrodynamic model, we observe significant changes with respect to the commonly employed local-response approximation, but also in comparison with homogeneous metal films where nonlocal effects have previously been considered. Notable are the emergence of a contribution from nonlocality at normal incidence and the surprisingly large structural parameters at which finite blueshifts are observable, which we attribute to diffraction that offers nonvanishing in-plane wave vector components and increases the penetration depth of longitudinal (nonlocal) modes.
\end{abstract}

DOI: 10.1103/PhysRevB.94.165410

\section{INTRODUCTION}

The increasing ability to fabricate particle arrays and holey metal films with nanoscale resolution has made the design and characterization of a wide range of nanostructured materials an attractive field to achieve new solutions towards total light control. Plasmonic crystals (PlCs) are intensely studied both in theory and experiment for their unique optical properties [1-9]: Hybrid photon-plasmon modes are found for corrugated metal films [10,11], nanoparticle arrays [12], photonic crystals supported by metal substrates $[13,14]$, and in a range of other periodic and aperiodic realizations.

Photons are strongly confined at the edge of band gaps in the crystal structure yielding strong local field enhancements. Plasmon-assisted enhancement of spontaneous and stimulated emission rates is crucial for the improvement of singlemolecule fluorescence and photoluminescence of quantum dots [15]. Such structures can achieve unidirectional emission radiation through plasmon-induced transparency and photontunneling effects that are interesting for optical filters and light guidance through directed transmission [9,16-19].

Dealing with mesoscopic metal structures makes it necessary to account for the quantum nature of electrons and include effects induced by the free conduction band [20-24]. The common local-response approximation (LRA) of classical electrodynamics omits short-ranged electron-electron coupling, while spatial dispersion in metals leads to a sizable interaction distance [25].

The successful observation of such quantum effects [26-30] has fueled the interest in an accurate description of electrons in the nonlocal regime and electron dynamics in photonic systems was studied in recent years with semiclassical [21,22,25,31-34] and $a b$ initio methods [35-41]. There has naturally been a large emphasis on localized plasmons in subwavelength metallic nanostructures and basic planar geometries [42],

\footnotetext{
*chrida@fotonik.dtu.dk
}

while only few works have considered plasmons in periodic structures [43-47].

Our aim is to study the influence of spatial dispersion in metal components of two-dimensional (2D) PlCs to increase the understanding of the underlying physical phenomena and investigate the limiting regimes of structural parameters towards the LRA at the nanoscale. While we restrict our discussion to holey metal films, the presented theoretical framework is applicable to particle arrays as well.

In this work, we apply the hydrodynamic model $[48,49]$ to calculate the electric field $\vec{E}$ at a given frequency $\omega$. It describes the dynamics of the free electron plasma separately via the (linearized) Navier-Stokes equation, emphasizing a real-space formulation [21-24]. The induced current density

$$
\vec{j}^{\text {ind }}=\frac{i}{\omega+i \gamma_{p}}\left(\frac{\omega_{p}^{2}}{4 \pi} \vec{E}-\beta^{2} \nabla \rho^{\text {ind }}\right)
$$

couples to the electromagnetic wave equation as

$$
\nabla \times \nabla \times \vec{E}-k^{2} \varepsilon_{b} \vec{E}=\frac{4 \pi i k^{2}}{\omega} \vec{j}^{\text {ind }}
$$

and relates to the induced charge density $\rho^{\text {ind }}$ through the continuity equation $\nabla \vec{j}^{\text {ind }}=i \omega \rho^{\text {ind }}$. The wave number is $k=$ $\omega / c$ with $c$ being the speed of light. Properties of the material are captured in the plasmon frequency $\omega_{p}$ and damping $\gamma_{p}$ which reflects the lifetime of collective electron excitations, as well as in the dielectric background $\varepsilon_{b}$ and the interaction strength of the nonlocal electrons $\beta=\sqrt{3 / 5} v_{F}$, where $v_{F}$ is the Fermi velocity. Note that we use Gauss units throughout this work.

Extending established periodic photonic crystal concepts based on the rigorous plane-wave method and scattering matrix approach allows us to investigate generalized geometries - particle arrays and holey metal films - with nonlocality [50,51]. We observe significant changes with respect to the LRA, but also in comparison with homogeneous metal 

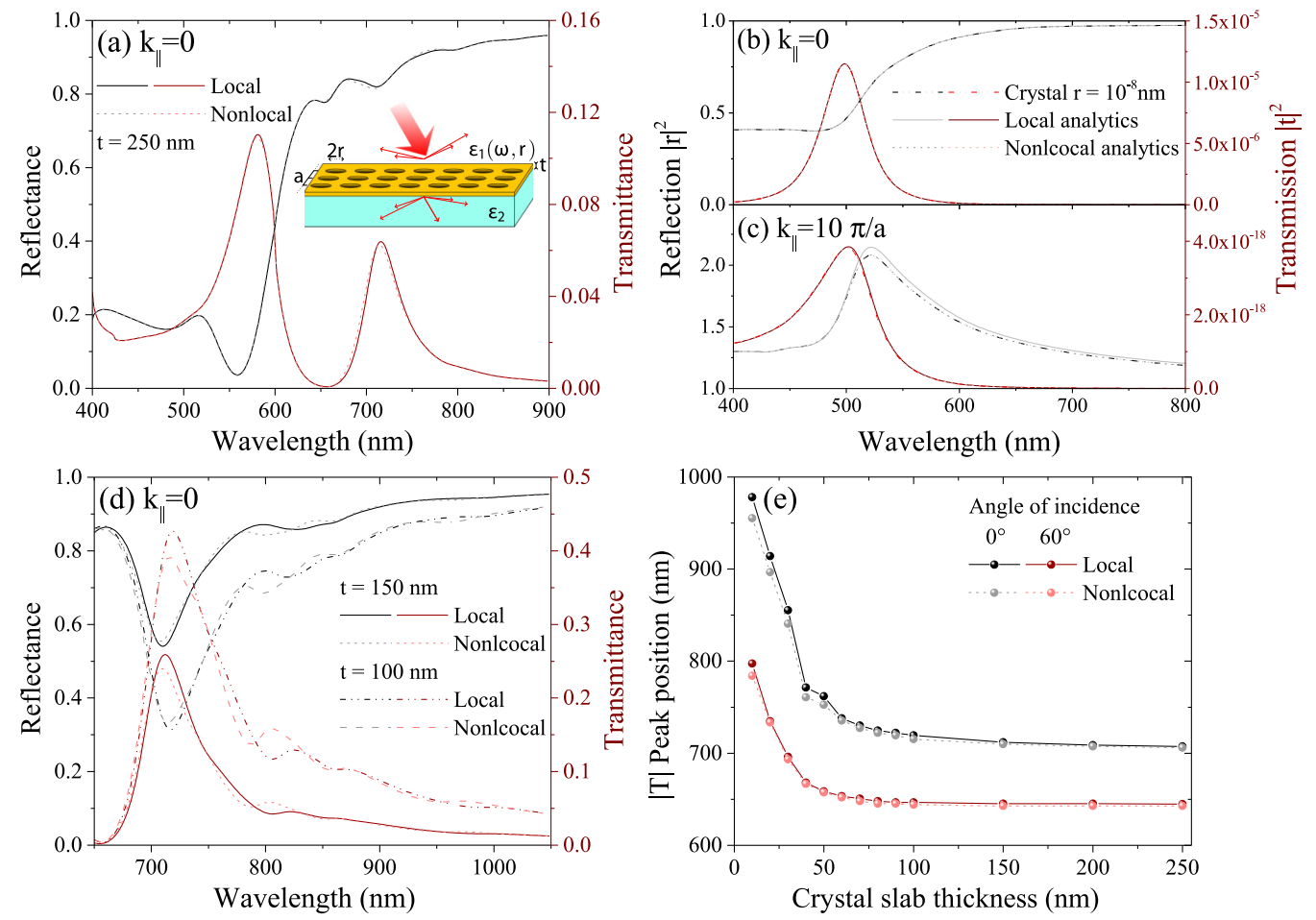

FIG. 1. (a) A holey metal film with thickness $t$ of space-modulated, frequency-dependent permittivity $\varepsilon_{1}$ (using bulk gold properties from tabulated experimental data) is investigated; see inset. The lattice constant is $a$ and separates air holes of radius $r$. A substrate with $\varepsilon_{2}$ supports the structure. The local crystal limit, see Refs. [16,17], with $a=400 \mathrm{~nm}, r=114.5 \mathrm{~nm}, t=250 \mathrm{~nm}$, and $\varepsilon_{2}=2.25$ is shown. At this film thickness, no nonlocal response is noted. (b), (c) For a symmetric environment $\left(\varepsilon_{1}=1\right)$ the homogeneous film limit $(r \rightarrow 0)$ is demonstrated; see Ref. [42]. (b) At vanishing parallel momentum local and nonlocal theory coincide (no longitudinal waves supported in a homogeneous film at $k_{\|}=0$ ). (c) For high enough parallel momentum, the homogeneous film shows nonlocal optical response. (d) Reducing the film thickness to $t=150 \mathrm{~nm}$ and $t=100 \mathrm{~nm}$ reveals resonance shifts and reduced intensities in the optical response induced by nonlocal effects in the crystal structure. (e) The film thickness is varied for two angles of incidence and the position of the lowest order transmittance peak is shown. Sizable resonance shifts are found for $t \leqslant 50 \mathrm{~nm}$.

films where nonlocal effects have been considered $[42,52,53]$. The most striking observations are the emergence of a contribution from spatial dispersion at normal incidence and the surprisingly large structural parameters at which small but finite blueshifts of resonances in the optical response are observable when compared to the spectral position predicted in the LRA. The former can be attributed to the diffraction the incoming light experiences due to the space-modulated crystal structure, which provides contributions from higher parallel momenta via $\vec{k}_{\|}^{\vec{G}}=\vec{k}_{\|}+\vec{G}=\left(k_{x}+n 2 \pi / a\right) \hat{x}+\left(k_{y}+m 2 \pi / a\right) \hat{y}$ in the plane-wave expansion. Hereby, reciprocal lattice vectors $\vec{G}$ define the diffraction order via the lattice period $a$ and integers $n, m$ where we assume a square lattice and contributions to the parallel momentum in the $x y$ plane. The latter effect can be seen as an interplay of a larger number of geometrical parameters important in a crystal that do not play any role in homogeneous films: not only film thickness, but also hole (or particle) size and lattice period become important. Moreover, when considering metal films with dielectric holes, larger holes provide narrow metal bridges or constrictions between different unit cells. From these results, a remarkable impact on high- $Q$ sensing applications, quantum dot emitters, molecular decay rates, and absorption spectra in large-area nanostructured materials is anticipated. A nonlocal method- ology for 2D PlCs can give a full account on the impact of nonlocal response for important concepts and applications in photonics, such as particle-array-enhanced spectroscopic methods [16-18,21,54-58] and transmission through holey metal films. We show that additional pressure waves induced by the electron oscillation lead to a change in the band structure and electromagnetic fields supported by a plasmonic crystal. This has previously been shown in 1D hyperbolic metamaterials and slit arrays [44-47] and in 2D periodic particle arrays exploiting the nonlocal polarizability of single particles [43].

The next section shows that the local (transversal) contribution to the solution of the coupled system does not observe any changes [50,51], but an additional, scalar wave equation for the longitudinal modes needs to be solved on which special emphasis is put. Eigenvalues and modes from the infinite crystal are then used in the scattering matrix technique to describe the case of a plasmonic crystal slab of finite thickness between two homogeneous semi-infinite dielectric regions; see inset in Fig. 1(a). Here, the complexity of the original local problem can be maintained and with it the iterative steps in propagating the scattering matrix. However, the field expressions and boundary conditions-extended by an additional boundary condition stemming from the 

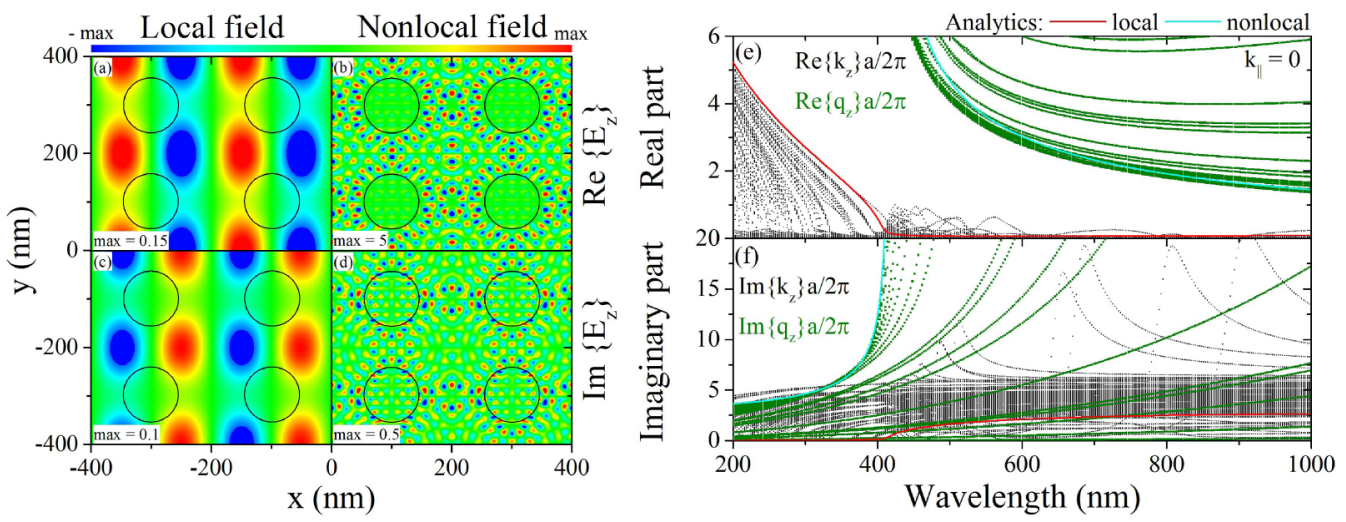

FIG. 2. (a)-(d) Example of reflected field distributions for a $t=150 \mathrm{~nm}$ crystal at normal incidence and $\lambda=660 \mathrm{~nm}$ (reflection maxima). (a), (b) Real and (c), (d) imaginary parts of the $z$ component of the electric field associated with the (a), (c) reflected local field, just outside of the front interface of the crystal, and (b), (d) nonlocal contributions calculated from Eq. (9b) just inside the crystal. (e) Real and (f) imaginary part of the eigenvalues $k_{z}$ (local) and $q_{z}$ (nonlocal) of the respective wave equations in comparison to the homogeneous thin film analytics. The crystal structure allows for a large penetration depth of nonlocal contributions (provision of modes with strongly reduced imaginary part) which makes nonlocal effects more prominent in crystal structures than in homogeneous films.

Navier-Stokes equation-become more involved leading to complex interface matrices. Such technicalities are laid out in detail in the appendices.

\section{PROPERTIES OF THE INFINITE CRYSTAL}

In this section, we briefly summarize the plane-wave expansion (PWE) method for both local (transversal) and nonlocal (longitudinal) solutions of the electromagnetic field in a 2D photonic crystal in the $x y$ plane with constant material properties in the $z$ direction. We follow Refs. [50,51] closely, introduce the PWE for all material parameters $\psi(\omega, \vec{r})=$ $\sum_{\vec{G}} e^{i \vec{G} \vec{R}} \hat{\psi}_{G}$, and use the Fourier transformation (FT) of the periodic real-space functions to compute matrices of dimensions $N \times N$ :

$$
\hat{\psi}_{G G^{\prime}}=\frac{1}{a^{2}} \int_{\mathcal{A}} \psi(\omega, \vec{r}) e^{-i\left(\vec{G}-\vec{G}^{\prime}\right) \vec{R}} d^{2} r
$$

where $\mathcal{A}$ denotes the unit cell area, $a$ is the periodicity of the square lattice, and $N$ is the integer giving the total number of reciprocal lattice vectors used as $N^{2}$ (different plane waves), which is a crucial parameter to achieve numerical convergence. Note that always $|n|,|m| \leqslant(N-1) / 2$. In contrast to the local case, not only the transversal permittivity $\varepsilon_{\perp}(\omega, \vec{r})=$ $\varepsilon_{b}(\omega, \vec{r})-\omega_{p}^{2} / \omega\left(\omega+i \gamma_{p}\right)$ but also further parameters such as the strength of the nonlocal interaction $\beta$ and the plasma frequency $\omega_{p}$ are modulated across the 2D crystal. All those parameters are step functions in real space and the FT for circular particles or holes is analytical [59].

Similarly, the electric and magnetic fields are expanded, accounting for the parallel momentum $\vec{k}_{\|}=\left(k_{x}, k_{y}\right)^{T}$ of the external field and a wave number $k_{z}\left(q_{z}\right)$ for the wave propagation in the $z$ direction for the local (nonlocal) contributions. With this, the classical (local) wave equation for the in-plane magnetic field results in $[50,51]$

$$
k_{z}^{2} \vec{\Phi}=\left\{\mathcal{E}\left[(\omega / c)^{2} I_{2 N^{2}}-\kappa\right]-K\right\} \vec{\Phi}
$$

with eigenvalues $k_{z}^{2}$, eigenmodes $\vec{\Phi}=\left(\Phi_{x}, \Phi_{y}\right)^{T}$, and furthermore $\mathcal{E}=\hat{\varepsilon}_{\perp} I_{2}$,

$K=\left(\begin{array}{ll}\hat{k_{x}} \hat{k_{x}} & \hat{k_{x}} \hat{k_{y}} \\ \hat{k_{y}} \hat{k_{x}} & \hat{k_{y}} \hat{k_{y}}\end{array}\right), \quad \kappa=\left(\begin{array}{cc}\hat{k_{y}} \hat{\varepsilon}_{\perp}^{-1} \hat{k_{y}} & -\hat{k_{y}} \hat{\varepsilon}_{\perp}^{-1} \hat{k_{x}} \\ -\hat{k_{x}} \hat{\varepsilon}_{\perp}^{-1} \hat{k_{y}} & \hat{k_{x}} \hat{\varepsilon}_{\perp}^{-1} \hat{k_{x}}\end{array}\right)$,

with diagonal matrices $\hat{k}_{\xi_{G G^{\prime}}}=\delta_{G G^{\prime}}\left(k_{\xi}+G_{\xi}\right)$, where in the $2 \mathrm{D}$ square crystal $\vec{G}=\frac{2 \pi}{a}(n \vec{x}+m \vec{y})$. The electric field follows from the previous solutions through

$$
M_{00} \equiv\left[(\omega / c)^{2} I_{2 N^{2}}-\kappa\right] \Phi\left(k_{z} \omega / c\right)^{-1} .
$$

As the nonlocal contributions are pressure-like waves of longitudinal character, they only add to the electric field, but leave the magnetic field unchanged [21,23], i.e., $\vec{e}_{\|}=\left(-e_{y}, e_{x}\right)^{T}=\vec{e}_{\| 0}+\vec{e}_{\| \mathrm{n} l}, \vec{h}_{\|} \equiv \vec{h}_{\| 0}$. The nonlocal wave equation is thus independent from the local contributions and results from the divergence of the induced current density $\vec{j}$ ind, Eq. (1), where the continuity equation $i \omega \rho^{\text {ind }}=\nabla \vec{j}$ ind is used and after introducing the PWE we obtain

$$
q_{z}^{2} \hat{\rho}=\left[\left(\hat{\beta}^{2}\right)^{-1} \omega\left(\omega+i \hat{\gamma}_{p}\right) \hat{\varepsilon}_{\perp} \hat{\varepsilon}_{b}^{-1}-\hat{k}_{x} \hat{k}_{x}-\hat{k}_{y} \hat{k}_{y}\right] \hat{\rho} .
$$

From Eqs. (2) and (1) we find the relevant matrix for nonlocal contributions $[42,53]$

$$
N_{00} \equiv-4 \pi i\left[\hat{\varepsilon}_{\perp} \omega\left(\omega+i \hat{\gamma}_{p}\right)\right]^{-1} \hat{\beta}^{2}\left(\begin{array}{c}
-\hat{k}_{y} \\
\hat{k}_{x}
\end{array}\right) \hat{\rho} .
$$

Both expressions $\left(M_{00}, N_{00}\right)$ are key ingredients to compute the optical properties of the finite crystal slab which is shown in more detail in the next section.

From $N_{00}$ it can be seen that-for vanishing parallel momentum - a homogeneous metal film does not support these types of pressure waves, but a nanostructured surface allowing for multiple diffraction orders is able to sustain such modes even for normal incident light. This is a striking difference from previous results for nonlocal but homogeneous thin films [42,53]. While we concentrate on studying the impact of nonlocality on finite crystal slabs, results for eigenvalues $k_{z}$ and $q_{z}$ of the infinite crystal are presented in Figs. 2(e) and 2(f). This allows drawing conclusions about the 
observed nonlocal modes also in contrast to results found for homogeneous (nonlocal) films and is discussed further below.

\section{OPTICAL PROPERTIES OF THE FINITE CRYSTAL}

With the modes and wave vectors calculated for the crystal layer, we are now ready to tackle the finite slab supported on a substrate [50]. The fields are in each layer expanded as

$$
\begin{aligned}
\vec{h}_{l, \|} & =\vec{\Phi}_{l}\left(f_{k l}(z) \vec{a}_{l}+f_{k l}(d-z) \vec{b}_{l}\right), \\
\vec{\rho}_{l} & =\hat{\rho}_{l}\left(f_{q l}(z) \vec{a}_{l, \mathrm{nl}}+f_{q l}(d-z) \vec{b}_{l, \mathrm{n} l}\right),
\end{aligned}
$$

where $\vec{\Phi}_{l}$ and $\hat{\rho}_{l}$ are the matrices containing the (local) magnetic and (nonlocal) charge density eigenvectors obtained in the previous section. The diagonal matrices $f_{\xi l}(z)_{n n^{\prime}}=$ $\delta_{n n^{\prime}} \exp \left(i \xi_{l, z n} z\right)$ are propagators composed of the respective eigenvalues $k_{l, z n}, q_{l, z n}$ and $\vec{a}_{l}, \vec{b}_{l}, \vec{a}_{l, \mathrm{nl}}, \vec{b}_{l, \mathrm{nl}}$ are the forward and backward scattering coefficients within each region.

\section{A. Local case}

The boundary conditions are given by the continuity of in-plane field components which can be written in a compact way for each layer as

$$
\left(\begin{array}{l}
\vec{e}_{\|, l} \\
\vec{h}_{\|, l}
\end{array}\right)=\mathcal{M}_{l}\left(\begin{array}{c}
f_{k l}(z) a_{l} \\
f_{k l}(d-z) b_{l}
\end{array}\right), \mathcal{M}_{l}=\left(\begin{array}{cc}
M_{l, 00} & -M_{l, 00} \\
\Phi_{l} & \Phi_{l}
\end{array}\right) .
$$

An analytical inverse of the block matrix $\mathcal{M}_{l}$ exists and the interface matrix is found in a straightforward manner:

$$
\begin{aligned}
I_{\mathrm{loc}}^{l, l+1}= & \mathcal{M}_{l}^{-1} \mathcal{M}_{l+1}=\frac{1}{2} \mathcal{M}_{l, 00}^{-1} \mathcal{M}_{l+1,00}\left(\begin{array}{rr}
1 & -1 \\
-1 & 1
\end{array}\right) \\
& +\frac{1}{2} \Phi_{l}^{-1} \Phi_{l+1}\left(\begin{array}{ll}
1 & 1 \\
1 & 1
\end{array}\right) .
\end{aligned}
$$

The scattering matrix for the cross-layer field propagation follows an iterative scheme to propagate solutions [50].

\section{B. Nonlocal case}

Transversal and longitudinal fields are summed up

$$
\begin{aligned}
\left(\begin{array}{l}
\vec{e}_{\|, l} \\
\vec{h}_{\|, l}
\end{array}\right) & =\mathcal{M}_{l}\left(\begin{array}{c}
f_{k l}(z) a_{l} \\
f_{k l}(d-z) b_{l}
\end{array}\right)+\mathcal{N}_{l}\left(\begin{array}{c}
f_{q l}(z) a_{l, \mathrm{nl}} \\
f_{q l}(d-z) b_{l, \mathrm{nl}}
\end{array}\right), \\
\mathcal{N}_{l} & =\left(\begin{array}{cc}
N_{00} & N_{00} \\
0 & 0
\end{array}\right) .
\end{aligned}
$$

A full solution requires $4 \times 4$ block matrices and two additional boundary conditions for the induced charge and current to be continuous, in order to solve for the four unknowns involved here. However, we can strongly simplify the calculation when we evoke the additional boundary condition first. This poses a few limitations to our approach. This and the widely analytic solution to the additional boundary condition are discussed in more detail in Appendix A. With the result obtained there in mind, we rewrite

$$
\left(\begin{array}{c}
\vec{e}_{\|, l} \\
\vec{h}_{\|, l}
\end{array}\right)=\mathcal{M}_{l}^{\star}\left(\begin{array}{c}
f_{k l}(z) a_{l} \\
f_{k l}(d-z) b_{l}
\end{array}\right)
$$

with an extended matrix $\mathcal{M}_{l}^{\star}$. This allows us to maintain the scattering matrix scheme [on extracting $f_{k l}(z)$ and $f_{k l}(d-z)$ from the parameters] and we can still find the analytic inverse of $\mathcal{M}_{l}^{\star}$. The expressions found are given in Appendix B.

The final scattering matrix contains the reflection and transmission coefficients which for the comparison to local results $[16,17]$ in Fig. 1 are further used to calculate the power flux (time-averaged Poynting vector) normalized to the power of the incident wave, i.e., reflectance and transmittance, in accordance to Ref. [51]. In these cases, due to the symmetry of the setup the polarization does not play a role. However, we also consider setups with finite parallel momentum where the incident field is evanescent and does not provide a suitable normalization factor since its power flux vanishes and we consider the optical coefficients directly. Moreover, the polarization does make an impact and we use $p$ polarization with $\vec{k}_{\|} \| \vec{x}$.

Note furthermore that the number of plane waves used $\left(N^{2}\right)$ ranges from $N=23\left(N^{2}=529\right)$ for the near-field plots in Fig. 2 at a specific wavelength to typically $N=31\left(N^{2}=961\right)$ for the calculated spectra. The convergence was checked for several spectra using up to $N=47\left(N^{2}=2209\right)$.

\section{RESULTS AND DISCUSSION}

The inset in Fig. 1(a) depicts the setup of the finite plasmonic crystal - here a holey metal (Au) film. In Figs. 1(b) and 1(c) we compare the developed theory in the homogeneous limit (vanishing hole size $r \rightarrow 0$ ) with analytic theory [42] for two cases of parallel momentum that result in local response in (b) and nonlocal response in (c). Note that for the homogeneous film the power flux of the incoming light vanishes for excitation with an evanescent source in Fig. 1(c), where coupling to higher diffraction orders is not possible, so that we compare the resulting reflection and transmission coefficients directly. Comparing to previous theoretical results on plasmonic crystals, Refs. [16,17], Fig. 1(a) reveals that these earlier studies are within the local-response regime as both local and nonlocal descriptions coincide. However, on decreasing the film thickness in the otherwise unchanged setup from $t=250 \mathrm{~nm}$ to $t=150 \mathrm{~nm}$ and $t=100 \mathrm{~nm}$, we begin to observe differences in the spectra of $2 \mathrm{~nm}$ and $4 \mathrm{~nm}$, respectively. Those are in agreement with previous findings on nonlocal interaction; that is, we observe a blueshift in the transmission peak (and reflection dip) positions as well as a reduction of the peak magnitudes (increase in the dips, resp.). The most striking aspect here is the fact that we consider normal incidence, which in the case of a homogeneous film always results in a suppression of longitudinal waves which need a finite value in the parallel momentum to emerge; cf. Fig. 1(b).

The crystal structure does, however, provide modes of higher order with finite in-plane components in the wave vector through $G_{\xi}=k_{\xi}+n 2 \pi / a$ which become larger for smaller $a$ and we expect an increasing influence on decreasing the 

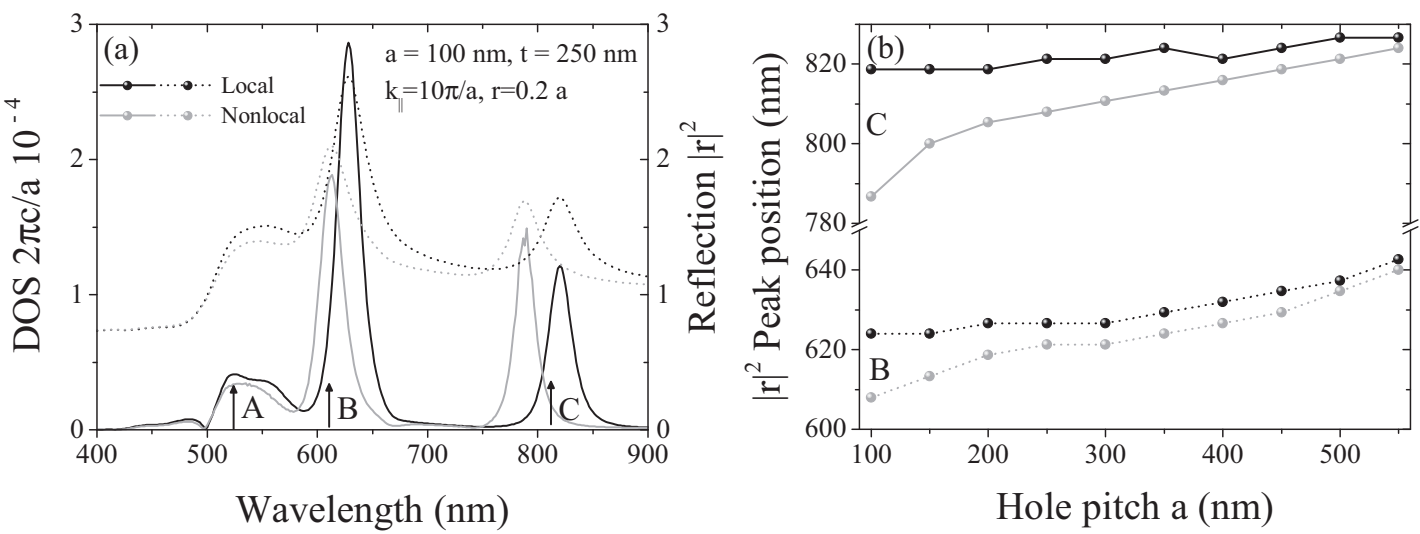

FIG. 3. Comparing the (a) reflection and corresponding density of optical states spectra and (b) the shift in peak positions as a function of hole separation for local and nonlocal plasmonic crystals using $k_{\|}=10 \pi / a, r=0.2 a, t=250 \mathrm{~nm}$ and in (a) for constant $a=100 \mathrm{~nm}$ for experimental data of gold. The reflected modes B and $\mathrm{C}$ are strongly impacted in the nonlocal theory at this high parallel momentum.

distance between the air holes (see for instance Fig. 3 discussed further below). The crystal readily provides several parameters of interest that could switch the properties from local to nonlocal. Interestingly, decreasing the hole size does yield the homogeneous film limit for vanishing hole sizes, so that indeed larger dielectric holes result in a stronger nonlocal response. For a hole radius close to the maximum of $r=0.5 a$ thin metal bridges are left between the neighboring holes and we conclude that strong nonlocal effects in holey metal films stem thus from fields scattered at the edges of the dielectric holes and longitudinal fields arising in thin metal walls confined by these holes. The film thickness plays a similar role to that for homogeneous nonlocal films; i.e., the thinner the crystal the stronger the observed resonance shifts as shown in Figs. 1(d) and $1(\mathrm{e})$.

We show near-field maps of the real and imaginary part of the $E_{z}$ component of the local field and the nonlocal contribution stemming from Eq. (8) (i.e., not the total field) in Figs. 2(a)-2(d) calculated from the optical coefficients at the air-crystal interface. Most strikingly, the wavelength of the longitudinal field is much smaller (about one order of magnitude) than that of the transversal field which is reflected in the nodes of standing waves seen in the near-fields. This can be understood by comparing the wave vectors-which are eigenvalues to the crystal structure-from the two wave equations, which we do in Figs. 2(e) and 2(f).

Let us first consider the case of a homogeneous metal film, where Eq. (4) reduces to $k_{z}=\sqrt{(\omega / c)^{2} \epsilon_{\perp}-k_{\|}^{2}}$, while the nonlocal wave number Eq. (7) becomes $q_{z}=$ $\sqrt{\epsilon_{\perp} /\left(\beta^{2} \epsilon_{b}\right) \omega(\omega+i \gamma)-k_{\|}^{2}}$. Assuming vanishing parallel momentum and damping, as well as for the sake of argument a Au metal film described by a Drude model with constant $\epsilon_{b}=9$, the remaining values allow a direct comparison to the expected analytic value through $\sqrt{(c / \beta)^{2} / \epsilon_{b}} \approx 90$. This means, the local and nonlocal wave numbers differ by a large factor in quantity, but are similar in their spectral structure. This remains true including a finite damping and parallel momentum accounting for the reciprocal vectors. This yields closely spaced dispersion relations of the different modes with a typical square root dependence, which can be seen in both
Figs. 2(e) and 2(f). However, the full solution of the complex eigenequations leads to further modes with no analogies to the homogeneous metal film; e.g., in Fig. 2(f) local modes are seen that have a spectral resonance structure, with a strongly increased imaginary part compared to the analytic homogeneous metal film (reflecting evanescent modes).

The real part of these eigenvalues determines the propagation constant, and the (smallest) nonlocal wave vector $q_{z}$ is indeed about one order of magnitude larger then the (largest) local $k_{z}=2 \pi / \lambda$ at the wavelength of incidence.

Another interesting conclusion can be drawn from the imaginary part in Fig. 2(f): Its inverse relates to the penetration depth of the wave, which becomes very small in the analytic case. For the crystal, the imaginary part becomes strongly reduced for a number of crystal modes which allows the longitudinal wave to penetrate more deeply into the structure. Thus we find a crossing of local and nonlocal modes which allows such excitations to exist simultaneously in the crystal with comparable penetration depths and hence contribution to the total field. This could explain why we see a significant resonance shift induced by nonlocal response at already rather thick slabs in both reflectance and transmittance; see Fig. 1(d).

We study one example of an incoming field with high parallel momentum far beyond the first Brillouin zone in Fig. 3. Due to the large value of the in-plane component of the wave vector, transmission is strongly reduced in the vertical direction. A spectrum for parameters comparable to the setup in Fig. 1(a) is given in Fig. 3(a), while 3(b) shows the dependence of the identified reflective modes (discussed further below) with the lattice period (while the radius is scaled as $r=0.2 a$ ). Next to the reflection $|r|^{2}$ in Fig. 3(a) we show the derived density of optical states (DOS) [61], which emphasizes the physical meaning of the observed resonances. For large parallel momentum, the solutions outside the metal slab are evanescent and the plasmon modes are consequently nonradiative.

At this high parallel momentum a clear difference between local and nonlocal modes is given, the observed blueshift with respect to the spectral position of the modes predicted in the LRA increases on decreasing the hole-hole separation, while both theories converge for larger hole distances. A strong 

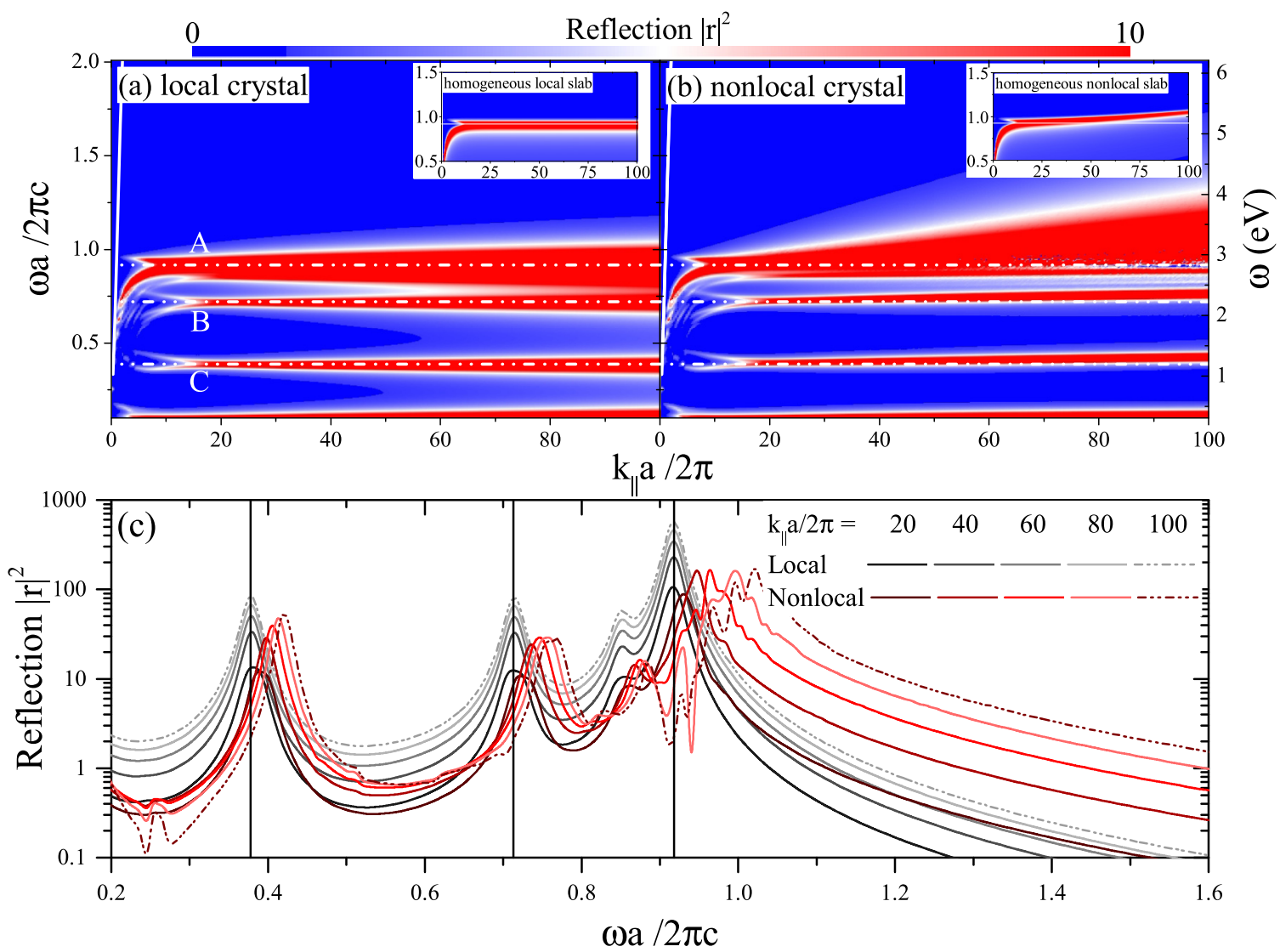

FIG. 4. Band structure calculated from the reflection coefficient $|r|^{2}$ for the (a) local and (b) nonlocal crystal with a Drude model for Au $\left(\epsilon_{b}=9\right)$ for clarity, $t=10 \mathrm{~nm}$, and other parameters as in Fig. 1(d). Corresponding homogeneous slab calculations are shown as insets. The guiding lines indicate the nondispersive local modes seen in (a). These modes correspond to the ones found in Fig. 3 for experimental data of gold, whereby mode A is suppressed with the fully complex background permittivity.

impact of longitudinal waves that are not present in the local theory of classical electrodynamics is thus demonstrated. This example shows very clearly the effects of the presence of nonlocal modes even at rather large structural parameters. While the feasibility of such a setup is disputable, decreasing geometrical sizes and using a lower parallel momentum coupled into the system can yield sizable spatial dispersion effects for an angle of incidence still within the first Brillouin zone, as was shown in Fig. 1(e).

Finally, we calculate the band structure from the reflectance in Fig. 4. In panels (a) and (b) we compare the local and nonlocal crystal's optical response as a function of the parallel momentum where the same reflection modes as in Fig. 3 are found. The insets show the corresponding homogeneous case and allow identifying mode $\mathrm{A}$ as the usual surface plasmon polariton present at the front surface of a homogeneous metal film. The higher order modes $\mathrm{B}$ and $\mathrm{C}$ emerge from reflection outside of the first Brillouin zone. Scattering into higher diffraction orders costs a specific amount of energy, so that we see those modes at lower energies. More modes appear when, e.g., decreasing the hole separation. Similar to findings for homogeneous slabs [42,52], the main optical mode does not saturate at a fixed frequency, but continues to shift. The guiding lines depict these fixed frequencies from the corresponding local modes. In the nonlocal case all those modes are blueshifted, but only the zeroth-order mode observes a significant continued shift with increasing in-plane component. The plasmon broadening is strongly increased as well. In Fig. 4(c), we directly compare specific spectra from (a) and (b).

\section{CONCLUSIONS}

For a plasmonic crystal placed in a dielectric environment, an extension of an existing rigorous plane-wave method accounting for nonlocal response in the metal components of the crystal could be achieved that maintains the complexity of the original problem. Hereby, the additional boundary condition stemming from the hydrodynamic equation for the free electron gas was exploited in an analytic way. An additional but scalar eigenvalue equation has to be solved, and the interface matrices used in the layer-by-layer propagation (scatteringmatrix theory) become more involved. It was demonstrated that nonlocal response plays a role already at normal incidence for nanostructured metal films whereby diffraction plays a major role in offering in-plane components in the wave vector not present in the incoming wave. Furthermore, a number of geometrical parameters influences the impact of nonlocality in these systems: (i) decreasing film thickness and (ii) increasing hole size in accordance to what can be expected from homogeneous films, as well as (iii) decreasing 
hole separation and (iv) thin metal walls confined between dielectric holes.

This study does exclude surface effects such as the electron spill-out $[40,53,60]$. However, as far as such effects can be attributed to a smooth change in the permittivity of the material this method can as well be used since a central ingredient is the Fourier transform of material parameters, which can be evaluated numerically for generalized surfaces. Accounting for a metal and thus possibly nonlocal substrate faces the challenges outlined in the appendices: A full extension would need a more complex matrix system of four unknowns. The bottleneck here is in particular the inverse of the interface matrix which cannot immediately be obtained by analytic means. The presented theory allows us to study a wide range of hybrid photon-plasmon systems. We concentrated here on metal films with dielectric holes, but complementary metal particle arrays can be studied. Defects with either nonlocal properties (e.g., a missing air hole) or dielectric properties (e.g., a missing metal particle) are of further interest. Other metamaterial designs that can be studied within our approach are (random) metal alloys and nonlocal inhomogeneous composites.

\section{ACKNOWLEDGMENTS}

We acknowledge Sanshui Xiao for fruitful discussions. C.D. thanks the Deutsche Forschungsgemeinschaft (German Research Foundation) for financial support through a DFG research fellowship (No. 268910011). J.C. acknowledges financial support from the Danish Council for Independent Research and a Sapere Aude Grant (FTP No.12-134776). N.A.M. acknowledges support from the Danish Council for Independent Research (FNU No. 1323-00087). The Center for Nanostructured Graphene is sponsored by the Danish National Research Foundation, Project No. DNRF103.

\section{APPENDIX A: ADDITIONAL BOUNDARY CONDITION}

Treating the free conduction band electrons with the linearized hydrodynamic equation makes it necessary to consider an additional boundary condition (ABC) [21,22,24,42,44,62]. The component along the surface normal of the induced current density $\hat{n} \cdot \vec{j}^{\text {ind }} \equiv 0$ at the crystal slab interfaces when both neighboring layers are dielectric, i.e., local, and a trespassing of the electron current is prohibited in the limit of an exceedingly high work function of the metal. It should be noted that this assumption neglects the impact of the electron spill-out relevant to metals with more modest work functions $[40,53,60,63]$.

While this step introduces a noniterative aspect into the layer-by-layer solution of the scattering matrix approach [50], it reduces the computational effort enormously, since we can solve the $\mathrm{ABC}$ inside the crystal layer independently from electronic properties of neighboring layers. We show in this section that we can maintain the dimensionality of the original problem with this limitation, instead of solving a system of four unknowns (local and nonlocal forward and backward scattering amplitudes) which would make an extension of the scattering matrix approach to $4 \times 4$ matrices of block matrices necessary in each iteration step.
We start from Eq. (1) with $0=\hat{n} \cdot \vec{j}^{\text {ind }}=j_{z}^{\text {ind }}$ :

$$
0=\frac{\omega_{p}^{2}}{4 \pi} e_{z}-\beta^{2} \partial_{z} \rho^{\text {ind }}=\frac{\omega_{p}^{2}}{4 \pi} e_{z}^{0}-\frac{\varepsilon_{b}}{\varepsilon_{\perp}} \beta^{2} \partial_{z} \rho^{\text {ind }} .
$$

Note that we omit the layer index $l$ for clarity; all material parameters and field amplitudes relate to the central crystal layer. Inserting the $e_{z}^{0}$ component and expanding into the field amplitudes Eqs. (9), we summarize material parameters in the matrix

$$
\mathcal{L}=i(\hat{\rho})^{-1} \frac{1}{q_{z}}\left(\hat{\beta}^{2}\right)^{-1} \frac{\hat{\varepsilon}_{\perp}}{\hat{\varepsilon}_{b}} \frac{\hat{\omega}_{p}^{2}}{4 \pi k} \frac{1}{\hat{\varepsilon}_{\perp}}\left(-\hat{k}_{y} \quad \hat{k}_{x}\right) \Phi,
$$

and can write the $\mathrm{ABC}$ in a compact way

$$
\mathcal{L}\left(f_{k}(z) \vec{a}+f_{k}(d-z) \vec{b}\right)=f_{q}(z) \vec{a}_{\mathrm{nl}}-f_{q}(d-z) \vec{b}_{\mathrm{nl}} .
$$

We evaluate this for the two interfaces $z=0$ and $z=d$ and abbreviate $f_{\xi}=f_{\xi}(d)$,

$$
\begin{aligned}
& \mathcal{L}\left(\vec{a}+f_{k} \vec{b}\right)=\left(\vec{a}_{\mathrm{nl}}-f_{q} \vec{b}_{\mathrm{nl}}\right), \\
& \mathcal{L}\left(f_{k} \vec{a}+\vec{b}\right)=\left(f_{q} \vec{a}_{\mathrm{nl}}-\vec{b}_{\mathrm{nl}}\right),
\end{aligned}
$$

from which we can work out

$$
\begin{aligned}
& {\left[I_{N}-f_{q} f_{q}\right] \vec{a}_{\mathrm{nl}}=\left(\mathcal{L}-f_{q} \mathcal{L} f_{k}\right) \vec{a}+\left(\mathcal{L} f_{k}-f_{q} \mathcal{L}\right) \vec{b},} \\
& {\left[I_{N}-f_{q} f_{q}\right] \vec{b}_{\mathrm{nl}}=\left(f_{q} \mathcal{L}-\mathcal{L} f_{k}\right) \vec{a}+\left(f_{q} \mathcal{L} f_{k}-\mathcal{L}\right) \vec{b} .}
\end{aligned}
$$

The interface matrix needs to be adjusted to include the additional terms. This is outlined in the next appendix.

\section{APPENDIX B: INTERFACE MATRIX IN NONLOCAL CASE}

The extended interface matrix $\mathcal{M}_{l}^{\star}$ can be built by using Eqs. (A4) in Eq. (12) which yields the general, $z$-dependent expressions

$$
\begin{aligned}
M_{l, 00}^{\star}= & M_{l, 00}+N_{00}\left[I_{N}-f_{q} f_{q}\right]^{-1}\left[f_{q}(z)\left(\mathcal{L}-f_{q} \mathcal{L} f_{k}\right)\right. \\
& \left.+f_{q}(d-z)\left(f_{q} \mathcal{L}-\mathcal{L} f_{k}\right)\right]\left[f_{k}(z)\right]^{-1}, \\
M_{l, 01}^{\star}= & -\left\{M_{00}-N_{00}\left[I_{N}-f_{q} f_{q}\right]^{-1}\left[f_{q}(z)\left(\mathcal{L} f_{k}-f_{q} \mathcal{L}\right)\right.\right. \\
& \left.\left.+f_{q}(d-z)\left(f_{q} \mathcal{L} f_{k}-\mathcal{L}\right)\right]\left[f_{k}(d-z)\right]^{-1}\right\} .
\end{aligned}
$$

Two cases can occur: When the nonlocal crystal layer is the next $l+1$ layer, we simply find

$$
I^{l, l+1}=\mathcal{M}_{l}^{-1} \mathcal{M}_{l+1}^{\star}=\frac{1}{2} M_{l, 00}^{-1} N_{00}\left(\begin{array}{cc}
\chi & \xi \\
-\chi & -\xi
\end{array}\right)+I_{\mathrm{loc}}^{l, l+1}
$$

with the parameters

$$
\begin{aligned}
\chi & =\left[I_{N}-f_{q} f_{q}\right]^{-1}\left(\left[I_{N}+f_{q} f_{q}\right] \mathcal{L}-2 f_{q} \mathcal{L} f_{k}\right), \\
\xi & =\left[I_{N}-f_{q} f_{q}\right]^{-1}\left(\left[I_{N}+f_{q} f_{q}\right] \mathcal{L}-2 f_{q} \mathcal{L} f_{k}^{-1}\right) .
\end{aligned}
$$

When the nonlocal layer is the current layer $l$, the inverse of the extended matrix is needed. Due to our simplified approach of having the metal crystal layer between dielectric layers, this inverse is analytic:

$$
\left(\mathcal{M}_{l}^{\star}\right)^{-1}=\left(\begin{array}{cc}
\left(v_{l}\right)^{-1} & \left(v_{l}\right)^{-1} \mu_{l} \Phi_{l}^{-1} \\
-\left(v_{l}\right)^{-1} & {\left[I_{N}-\left(v_{l}\right)^{-1} \mu_{l}\right] \Phi_{l}^{-1}}
\end{array}\right),
$$


where $v_{l}=2\left[M_{l, 00}-N_{00}(\chi+\xi) / 2\right], \quad \mu_{l}=M_{l, 00}-N_{00} \chi$, and the interface matrix becomes

$$
\begin{aligned}
\left(\mathcal{M}_{l}^{\star}\right)^{-1} \mathcal{M}_{l+1}= & \left(v_{l}\right)^{-1} M_{l+1,00}\left(\begin{array}{cc}
1 & -1 \\
-1 & 1
\end{array}\right)+\Phi_{l}^{-1} \Phi_{l+1} \\
& \times\left(\begin{array}{cc}
\left(v_{l}\right)^{-1} \mu_{l} & \left(v_{l}\right)^{-1} \mu_{l} \\
I_{N}-\left(v_{l}\right)^{-1} \mu_{l} & I_{N}-\left(v_{l}\right)^{-1} \mu_{l}
\end{array}\right) .
\end{aligned}
$$

It should be noted that in the homogeneous case $N_{00} \mathcal{L}=$ $\frac{k_{z \perp}}{k \varepsilon_{\perp}} \frac{k_{\|}^{2}}{q k_{z \perp}}\left(1-\frac{\varepsilon_{\perp}}{\varepsilon_{b}}\right) \equiv \frac{k_{z 1}}{k \varepsilon_{\perp}} g$ as found previously [42] and with

$$
\begin{array}{r}
G^{ \pm}=\frac{g}{i \sin (q d)}\left[e^{ \pm i k_{z 1} d}-\cos (q d)\right], \text { e.g. }, \\
r_{p}=\frac{k_{z 0} \varepsilon_{\perp}-k_{z 1} \varepsilon_{0}\left(1-G^{-}\right)}{k_{z 0} \varepsilon_{\perp}+k_{z 1} \varepsilon_{0}\left(1+G^{+}\right)}, \\
t_{p}=\frac{2 k_{z 0} \varepsilon_{\perp}}{k_{z 0} \varepsilon_{\perp}+k_{z 1} \varepsilon_{0}\left(1+G^{+}\right)},
\end{array}
$$

for the front surface. The homogeneous analytics are used in Figs. 1(b) and 1(c), Figs. 2(e) and 2(f), and the insets in Figs. 4(a) and 4(b).
[1] T. W. Ebbesen, H. J. Lezec, H. F. Ghaemi, T. Thio, and P. A. Wolff, Nature (London) 391, 667 (1998).

[2] E. Popov, M. Neviere, S. Enoch, and R. Reinisch, Phys. Rev. B 62, 16100 (2000).

[3] W. L. Barnes, A. Dereux, and T. Ebbesen, Nature (London) 424, 824 (2003).

[4] W. L. Barnes, W. A. Murray, J. Dintinger, E. Devaux, and T. W. Ebbesen, Phys. Rev. Lett. 92, 107401 (2004).

[5] F. J. García-Vidal, L. Martín-Moreno, and J. B. Pendry, J. Opt. A: Pure Appl. Opt. 7, S97 (2005).

[6] P. Lalanne, J. P. Hugonin, and J. C. Rodier, Phys. Rev. Lett. 95, 263902 (2005).

[7] M. Beruete, M. Sorolla, and I. Campillo, Opt. Express 14, 5445 (2006).

[8] F. J. García de Abajo, Rev. Mod. Phys. 79, 1267 (2007).

[9] T. V. Teperik, F. J. García de Abajo, A. G. Borisov, M. Abdelsalam, P. N. Bartlett, Y. Sugawara, and J. J. Baumberg, Nat. Photonics 2, 299 (2008).

[10] J. A. Porto, F. J. García-Vidal, and J. B. Pendry, Phys. Rev. Lett. 83, 2845 (1999).

[11] T. A. Kelf, Y. Sugawara, J. J. Baumberg, M. Abdelsalam, and P. N. Bartlett, Phys. Rev. Lett. 95, 116802 (2005).

[12] G. Vecchi, V. Giannini, and J. Gómez Rivas, Phys. Rev. B 80, 201401 (2009).

[13] X. Yang, A. Ishikawa, X. Yin, and X. Zhang, ACS Nano 5, 2831 (2011).

[14] K. J. Russell, T.-L. Liu, S. Cui, and E. L. Hu, Nat. Photonics 6, 459 (2012).

[15] G. Vecchi, V. Giannini, and J. Gómez Rivas, Phys. Rev. Lett. 102, 146807 (2009)

[16] F. Przybilla, A. Degiron, J.-Y. Laluet, C. Genet, and T. W. Ebbesen, J. Opt. A: Pure Appl. Opt. 8, 458 (2006).

[17] S. G. Rodrigo, F. J. García-Vidal, and L. Martín-Moreno, Phys. Rev. B 77, 075401 (2008).

[18] L. Martín-Moreno, F. J. García-Vidal, H. J. Lezec, K. M. Pellerin, T. Thio, J. B. Pendry, and T. W. Ebbesen, Phys. Rev. Lett. 86, 1114 (2001).

[19] S. Feng and Y. Wang, Opt. Express 21, 220 (2013).

[20] J. Zuloaga, E. Prodan, and P. Nordlander, Nano Lett. 9, 887 (2009).

[21] C. David and F. J. García de Abajo, J. Phys. Chem. C 115, 19470 (2011).

[22] S. Raza, G. Toscano, A.-P. Jauho, M. Wubs, and N. A. Mortensen, Phys. Rev. B 84, 121412(R) (2011).

[23] N. A. Mortensen, S. Raza, M. Wubs, T. Søndergaard, and S. I. Bozhevolnyi, Nat. Commun. 5, 3809 (2014).
[24] S. Raza, S. I. Bozhevolnyi, M. Wubs, and N. A. Mortensen, J. Phys.: Condens. Matter 27, 183204 (2015).

[25] F. J. García de Abajo, J. Phys. Chem. C 112, 17983 (2008).

[26] J. A. Scholl, A. L. Koh, and J. A. Dionne, Nature (London) 483, 421 (2012).

[27] C. Ciracì, R. T. Hill, J. J. Mock, Y. Urzhumov, A. I. FernándezDomínguez, S. A. Maier, J. B. Pendry, A. Chilkoti, and D. R. Smith, Science 337, 1072 (2012).

[28] K. J. Savage, M. M. Hawkeye, R. Esteban, A. G. Borisov, J. Aizpurua, and J. J. Baumberg, Nature (London) 491, 574 (2012).

[29] S. Raza, N. Stenger, S. Kadkhodazadeh, S. V. Fischer, N. Kostesha, A.-P. Jauho, A. Burrows, M. Wubs, and N. A. Mortensen, Nanophotonics 2, 131 (2013).

[30] S. Raza, S. Kadkhodazadeh, T. Christensen, M. Di Vece, M. Wubs, N. A. Mortensen, and N. Stenger, Nat. Commun. 6, 8788 (2015).

[31] A. Wiener, A. I. Fernández-Domínguez, A. P. Horsfield, J. B. Pendry, and S. A. Maier, Nano Lett. 12, 3308 (2012).

[32] D. de Ceglia, S. Campione, M. A. Vincenti, F. Capolino, and M. Scalora, Phys. Rev. B 87, 155140 (2013).

[33] Y. Luo, A. I. Fernández-Domínguez, A. Wiener, S. A. Maier, and J. B. Pendry, Phys. Rev. Lett. 111, 093901 (2013).

[34] T. Christensen, W. Yan, S. Raza, A.-P. Jauho, N. A. Mortensen, and M. Wubs, ACS Nano 8, 1745 (2014).

[35] P. T. Leung, Phys. Rev. B 42, 7622 (1990).

[36] A. Liebsch, Phys. Rev. B 48, 11317 (1993).

[37] T. V. Teperik, P. Nordlander, J. Aizpurua, and A. G. Borisov, Phys. Rev. Lett. 110, 263901 (2013).

[38] K. Andersen, K. L. Jensen, N. A. Mortensen, and K. S. Thygesen, Phys. Rev. B 87, 235433 (2013).

[39] P. Zhang, J. Feist, A. Rubio, P. García-González, and F. J. GarcíaVidal, Phys. Rev. B 90, 161407 (2014).

[40] W. Yan, M. Wubs, and N. Asger Mortensen, Phys. Rev. Lett. 115, 137403 (2015).

[41] T. P. Rossi, A. Zugarramurdi, M. J. Puska, and R. M. Nieminen, Phys. Rev. Lett. 115, 236804 (2015).

[42] C. David, N. A. Mortensen, and J. Christensen, Sci. Rep. 3, 2526 (2013).

[43] V. Yannopapas, J. Phys.: Condens. Matter 20, 325211 (2008).

[44] W. Yan, M. Wubs, and N. A. Mortensen, Phys. Rev. B 86, 205429 (2012).

[45] A. Yanai, N. A. Mortensen, and U. Levy, Phys. Rev. B 88, 205120 (2013).

[46] A. Moreau, C. Ciracì, and D. R. Smith, Phys. Rev. B 87, 045401 (2013). 
[47] M. Dechaux, P.-H. Tichit, C. Ciracì, J. Benedicto, R. Pollès, E. Centeno, D. R. Smith, and A. Moreau, Phys. Rev. B 93, 045413 (2016).

[48] F. Bloch, Z. Phys. 81, 363 (1933).

[49] D. Pines and D. Bohm, Phys. Rev. 85, 338 (1952).

[50] D. M. Whittaker and I. S. Culshaw, Phys. Rev. B 60, 2610 (1999).

[51] V. Liu and S. Fan, Comput. Phys. Commun. 183, 2233 (2012).

[52] S. Raza, T. Christensen, M. Wubs, S. I. Bozhevolnyi, and N. A. Mortensen, Phys. Rev. B 88, 115401 (2013).

[53] C. David and F. J. García de Abajo, ACS Nano 8, 9558 (2014).

[54] H. F. Ghaemi, T. Thio, D. E. Grupp, T. W. Ebbesen, and H. J. Lezec, Phys. Rev. B 58, 6779 (1998).

[55] J. Zhang and C. Noguez, Plasmonics 3, 127 (2008).

[56] L. Rodríguez-Lorenzo, R. A. Álvarez-Puebla, I. PastorizaSantos, S. Mazzucco, O. Stéphan, M. Kociak, L. M. Liz-Marzán, and F. J. García de Abajo, J. Am. Chem. Soc. 131, 4616 (2009).
[57] J.-J. Feng, U. Gernert, M. Sezer, U. Kuhlmann, D. H. Murgida, C. David, M. Richter, A. Knorr, P. Hildebrandt, and I. M. Weidinger, Nano Lett. 9, 298 (2009).

[58] G. Toscano, S. Raza, S. Xiao, M. Wubs, A.-P. Jauho, S I. Bozhevolnyi, and N. A. Mortensen, Opt. Lett. 37, 2538 (2012).

[59] S. Guo and S. Albin, Opt. Express 11, 167 (2003).

[60] G. Toscano, J. Straubel, A. Kwiatkowski, C. Rockstuhl, F. Evers, H. Xu, N. A. Mortensen, and M. Wubs, Nat. Commun. 6, 7132 (2015).

[61] V. Gasparian, T. Christen, and M. Büttiker, Phys. Rev. A 54, 4022 (1996).

[62] A. Boardman and R. Ruppin, Surf. Sci. 112, 153 (1981).

[63] D. Jin, Q. Hu, D. Neuhauser, F. von Cube, Y. Yang, R. Sachan, T. S. Luk, D. C. Bell, and N. X. Fang, Phys. Rev. Lett. 115, 193901 (2015) 